\title{
VALUATIONS ON MEROMORPHIC FUNCTIONS OF BOUNDED TYPE
}

\author{
MITSURU NAKAI
}

\begin{abstract}
The primary purpose of this paper is to show that every valuation on the field of meromorphic functions of bounded type on a finitely sheeted unlimited covering Riemann surface is a point valuation if and only if the same is true on its base Riemann surface. The result is then applied to concrete examples and some related results are obtained.
\end{abstract}

Any valuation on the field $M(W)$ of single valued meromorphic functions on a Riemann surface $W$ is a point valuation [9]. What happens to valuations on subfields of $M(W)$ ? An especially interesting subfield in this context is the field $M^{\infty}(W)$ of meromorphic functions of bounded type on $W$ (cf. [2]). We are thus concerned with the following question in this paper: When is it true that any valuation on nontrivial $M^{\infty}(W)$ is a point valuation?

The paper consists of five parts. $\S 1$ covers preliminaries. The main part, $\S 2$, concerns the covering stability. We say that a Riemann surface $W$ is stable if $M^{\infty}(W)$ is nontrivial and any valuation on $M^{\infty}(W)$ is a point valuation. Then it is shown in this section as the main theorem of this paper that a finitely sheeted unlimited covering surface $R$ of a Riemann surface $S$ is stable if and only if $S$ is stable. In $\S 3$ on stable surfaces, an example due to Forelli [4] of stable surfaces is given among others. In $\S 4$ the relation between $H^{\infty}$-maximality and stability of a Riemann surface $W$ and in particular of a plane region $W$ is discussed. We relax the definition of stability in $\S 5$ to obtain the notion of weak stability of a Riemann surface $W$. Here a simple but powerful device of what we call $H^{\infty}$-barrier is introduced, which is used to exhibit a weakly stable plane region of infinite connectivity.

\section{Preliminaries.}

1.1. Fields $F$ we consider in this paper are all assumed to be extensions of the complex number field $\mathbf{C}$. We denote by $F^{*}$ the multiplicative group consisting of nonzero elements in $F$, i.e. $F^{*}=F \backslash\{0\}$. By a valuation $v$ on $F$ we mean a discrete valuation $v$ on $F$, i.e. a group homomorphism of the multiplicative group $F^{*}$ into the additive group $\mathbf{Z}$ of integers such that

$$
v(f+g) \geq \min (v(f), v(g)) \quad\left(f, g \in F^{*}\right)
$$

where we make the convention that $v(0)=+\infty$.

Received by the editors June 16, 1987.

1980 Mathematics Subject Classification. Primary 30H50; Secondary 30F99, 30D50, 46J15.

To complete this work the author was supported in part by Grant-in-Aid for Scientific Research, No. 61540094, Japanese Ministry of Education, Science and Culture. 
As a consequence of the discreteness of a valuation $v$ it automatically follows that

$$
v\left(\mathbf{C}^{*}\right)=\{0\},
$$

i.e. $v(\lambda)=0$ for every $\lambda$ in $\mathbf{C}^{*}$. For the proof observe that $\lambda^{1 / n}$ exists in $\mathbf{C}^{*}$ for every $n$ in $\mathbf{N}$, the set of positive integers. Since $v(\lambda)=n v\left(\lambda^{1 / n}\right)$ means that the integer $v(\lambda)$ is divisible by any $n$ in $\mathbf{N}$, we must conclude that $v(\lambda)=0$.

It is important but easy to see that the equality holds in (1.1) if $v(f) \neq v(g)$. The following direct consequence of this will also be frequently made use of: $v(f+\lambda)=0$ for every $\lambda$ in $\mathbf{C}^{*}$ if $v(f)>0$.

We also assume that any valuation $v$ considered in this paper is nontrivial in the sense that the value group $v\left(F^{*}\right)$ of $v$ is different from the trivial subgroup $\{0\}$ of $\mathbf{Z}$. Then $v\left(F^{*}\right)=\{m e: m \in \mathbf{Z}\}$ where $e$ is the minimum of the set of positive numbers in $v\left(F^{*}\right)$. The value group $\hat{v}\left(F^{*}\right)$ of the normalized valuation $\hat{v}$ of $v$, referred to as the normalization of $v$, defined by

$$
\hat{v}(f)=e^{-1} v(f) \quad\left(f \in F^{*}\right)
$$

is the whole group $\mathbf{Z}$. Two valuations $v_{1}$ and $v_{2}$ on $F$ are said to be equivalent, $v_{1} \sim v_{2}$ in notation, if $\hat{v}_{1}=\hat{v}_{2}$. The set $\{f \in F: v(f) \geq 0\}$ is referred to as the valuation ring and $\{f \in F: v(f)>0\}$ the valuation ideal of $v$.

For valuations, see e.g. $[3,10,15,18]$, among many others.

1.2. We denote by $M(W)$ the field of single valued meromorphic functions on a Riemann surface $W$. Take a local parameter $z$ at a point $a$ in $W$ with $z(a)=0$. Let $f$ be defined and meromorphic in a neighborhood of $a$ and

$$
f(z)=\sum_{\nu=k}^{\infty} c_{\nu} z^{\nu} \quad\left(c_{k} \neq 0\right)
$$

be the Laurent expansion of $f$. If $f$ is not identically zero, then the number $k$ is uniquely determined by $f$ and $a$ not depending on the choice of $z$. The number $k$ is usually denoted by $\partial_{f}(a)$ and called the order of $f$ at $a[8]$. We conventionally set $\partial_{0}(a)=+\infty$.

We say that a subfield $F$ of $M(W)$ is nontrivial if $F \supset \mathbf{C}$ and $F \neq \mathbf{C}$. It is easily checked that the mapping $\partial .(a): f \mapsto \partial_{f}(a)$ is a valuation on any nontrivial subfield $F$ of $M(W)$. Any valuation $v$ on $F$ equivalent to $\partial$. (a) for an $a \in W$ is referred to as a point valuation on $F$ at $a$, i.e. $v$ is a point valuation if $v(f)=$ const $\partial_{f}(a)$ for every $f$ in $F^{*}$ with an $a$ in $W$.

It is well known that every valuation on the total field $M(W)$ is a point valuation. If $W$ is closed, then the result is classical, and if $W$ is open, then the result is known as the Iss'ssa theorem [9]. We will investigate the question to what extent the similar situation can occur if $M(W)$ is replaced by its subfield consisting of functions of bounded type.

1.3. A single valued meromorphic function $f$ on a Riemann surface $W$ is said to be of bounded type if $f=g / h$ on $W$ where $g$ and $h$ are bounded holomorphic functions on $W$ with $h \not \equiv 0$. We denote by $M^{\infty}(W)$ the field of single valued meromorphic functions of bounded type on $W$ so that $M^{\infty}(W)$ is a subfield of $M(W)$ containing $\mathbf{C}$ and also a quotient field of the algebra $H^{\infty}(W)$ of bounded holomorphic functions on $W$. Clearly $M^{\infty}(W)$ is nontrivial if and only if $H^{\infty}(W)$ is 
nontrivial in the sense that $H^{\infty}(W) \neq \mathbf{C}$ or $W \notin O_{A B}$ in the notation of classification theory of Riemann surfaces (cf. e.g. [14]).

For convenience we say that a Riemann surface $W$ is $H^{\infty}$-stable or simply stable if $M^{\infty}(W)$ is nontrivial and every valuation on $M^{\infty}(W)$ is a point valuation. Thus our problem is to clarify the stability of Riemann surfaces under consideration.

We remark here that any valuation $v$ on $M^{\infty}(W)$ is nonnegative on $H^{\infty}(W)$ (cf. [11]), i.e.

$$
v(f) \geq 0 \quad\left(f \in H^{\infty}(W)\right) .
$$

In fact, let $c$ be a positive number greater than $\sup _{W}|f|$. Then $(c+f)^{1 / n}$ belongs to $H^{\infty}(W)$ for any $n$ in $\mathbf{N}$ and the identity

$$
\left.n v\left((c+f)^{1 / n}\right)\right)=v(c+f)
$$

shows that the integer $v(c+f)$ is divisible by any $n$ in $\mathbf{N}$ so that $v(c+f)=0$. By (1.1) and (1.2) we have $v(f)=v(f+c-c) \geq \min (v(f+c), v(-c))=0$.

A valuation $v$ on $M^{\infty}(W)$ is said to be distinguished if the following condition is satisfied: If $v(f) \geq 0$ for an $f$ in $M^{\infty}(W)$, then there exists a $\lambda$ in $\mathbf{C}$ such that $v(f-\lambda)>0$. It is easy to see that such a $\lambda$ is uniquely determined by $f$. Point valuations clearly satisfy this condition and therefore point valuations are distinguished. Except for $\S 5$ we do not a priori assume the distinguishedness for our valuations in this paper which is one of the important points to be stressed in our study.

We say that $W$ is weakly $H^{\infty}$-stable or simply weakly stable if $M^{\infty}(W)$ is nontrivial and every distinguished valuation on $M^{\infty}(W)$ is a point valuation. The stability clearly implies the weak stability but the converse is not at all known to be valid or invalid. In this connection the following question is very important and interesting but seems to be hard to resolve:

OPEN PROBLEM 1. Is there any $W$ such that $M^{\infty}(W)$ carries a nondistinguished valuation or is any valuation on any $M^{\infty}(W)$ automatically distinguished?

1.4. We say that a Riemann surface $R$ or more precisely a triple $(R, S, \pi)$ of Riemann surfaces $R$ and $S$ and an analytic mapping $\pi$ of $R$ into $S$ is a covering surface of $S$. The surface $S$ and the mapping $\pi$ are referred to as the base surface and the covering map or projection of the covering surface $(R, S, \pi)$.

We say that the covering surface $(R, S, \pi)$ is unlimited if for any curve $C$ on $S$ with its initial point $a$ and any $\alpha$ in $\pi^{-1}(a)$ there exists a curve $\Gamma$ on $R$ with $\alpha$ its initial point such that $\pi(\Gamma)=C$.

Let $a$ be in $S$ and $\alpha$ in $\pi^{-1}(a)$. We can always find local parameters $z$ and $\varsigma$ about $a$ and $\alpha$ respectively such that the local expression of the covering map $z=\pi(\varsigma)$ takes the form $z=\varsigma^{m}$. Here the positive integer $m$, the multiplicity of $\alpha$, does not depend on the choice of local parameters $z$ and $\varsigma$. If $m>1$, then $\alpha$ is referred to as a branch point of order $m-1$.

For each $a$ in $S$ we let $\#\left(\pi^{-1}(a)\right)=\infty$ if $\pi^{-1}(a)$ is an infinite set and $\#\left(\pi^{-1}(a)\right)$ $=n$ if the set $\pi^{-1}(a)$ consists of a finite $n$ number of points where a branch point of order $m-1$ is counted as $m$ points.

When $(R, S, \pi)$ is unlimited, \#( $\left.\pi^{-1}(a)\right)$ is a constant $n$ in $\mathbf{N} \cup\{\infty\}$ for every $a$ in $S$. If $n \in \mathbf{N}$, then we say that $(R, S, \pi)$ is $n$ sheeted or more roughly finitely 
sheeted without specifying $n$ or more simply finite. Note that there may or may not be an infinite number of branch points in $R$ even for unlimited finite covering surfaces $R$.

\section{The covering stability.}

2.1. The final goal of the study of stability should be to completely determine the family of stable Riemann surfaces. Examples of nonstable Riemann surfaces are in plenty. However only a few surfaces are known to be stable and the first step to the above goal is to try to enlarge the class of known examples of stable surfaces. The primary purpose of this paper is to contribute to this first step by supplying a device to enrich the class of known examples of stable surfaces. The following gives this device and is the main result of this paper.

THEOREM 1. The unlimited finite covering surface $R$ is stable if and only if its base Riemann surface $S$ is stable.

The result above also holds and actually is easier to see to hold if the stability is replaced by the weak stability. Once more we stress here that the importance of the above result lies in the fact that we do not assume the distinguishedness of valuations in advance. The proof of the above theorem will be given in subsections $2.2-2.8$.

2.2. We consider an $n$ sheeted covering surface $(R, S, \pi)$ with an $n$ in $\mathbf{N}$. We will show that $R$ is stable if and only if $S$ is stable. Identifying $M^{\infty}(S) \circ \pi$ with $M^{\infty}(S)$ we may consider $M^{\infty}(S) \subset M^{\infty}(R)$, i.e. $M^{\infty}(S)$ is a subfield of $M^{\infty}(R)$, if no confusion would arise. The characteristic of $M^{\infty}(S)$, as a field extension of $\mathrm{C}$, is of course zero and therefore $M^{\infty}(R) / M^{\infty}(S)$ is a separable extension.

To clarify the dependence of the field extension $M^{\infty}(R)$ on $M^{\infty}(S)$ it is customary to consider fundamental symmetric functions $c_{1}, \ldots, c_{n}$ on $S$ given by

$$
(-1)^{k} c_{k}(z)=\sum_{i_{1}<\cdots<i_{k}}^{1, \ldots, n} f\left(z_{i_{1}}\right) \cdots f\left(z_{i_{k}}\right) \quad(k=1, \ldots, n)
$$

for functions $f$ in $M(R)$ with

$$
\pi^{-1}(z)=\left\{z_{1}, \ldots, z_{n}\right\} \quad(z \in S)
$$

where a branch point of order $m-1$ is appearing in the above as $m$ points. Functions $c_{1}, \ldots, c_{n}$ are seen to belong to $M(S)$ and will be referred to as $c$-functions associated with $f$. and

If $f=g / h$ with $g$ and $h$ in $H^{\infty}(R)$, then $c_{k}=d_{k} / e$ with $e(z)=h\left(z_{1}\right) \cdots h\left(z_{n}\right)$

$$
(-1)^{k} d_{k}(z)=\sum_{i_{1}<\cdots<i_{k}}^{1, \ldots, n} g\left(z_{i_{1}}\right) \cdots g\left(z_{i_{k}}\right) h\left(z_{j_{1}}\right) \cdots h\left(z_{j_{n-k}}\right)
$$

where $\left\{j_{1}, \ldots, j_{n-k}\right\}=\{1, \ldots, n\} \backslash\left\{i_{1}, \ldots, i_{k}\right\}$. Hence

$$
\|e\|_{S} \leq\|h\|_{R}^{n}, \quad\left\|d_{k}\right\|_{S} \leq\left(\begin{array}{l}
n \\
k
\end{array}\right)\|g\|_{R}^{k}\|h\|_{R}^{n-k}
$$

where $\|u\|_{X}=\sup _{X}|u|$ for any function $u$ on a set $X$. Therefore $c_{k} \in M^{\infty}(S)$ $(k=1, \ldots, n)$ along with $f \in M^{\infty}(R)$ and in particular $c_{k} \in H^{\infty}(S)(k=1, \ldots, n)$ if $f \in H^{\infty}(R)$. 
From (2.1) it follows that

$$
f^{n}+c_{1} f^{n-1}+\cdots+c_{n-1} f+c_{n}=0 .
$$

This means that any $f$ in $M^{\infty}(R)$ is algebraic over $M^{\infty}(S)$ and

$$
\left[\left[M^{\infty}(S)\right](f): M^{\infty}(S)\right] \leq n
$$

for every $f$ in $M^{\infty}(R)$, where $[L: F]$ is the degree of a field extension $L / F$ and $F(x)$ is the extension field obtained from a field $F$ by adding a primitive element $x$.

As a consequence of $(2.3)$ we see that

$$
\left[M^{\infty}(R): M^{\infty}(S)\right] \leq n .
$$

If this were not the case, then there would exist a subfield $L$ between $M^{\infty}(S)$ and $M^{\infty}(R)$ with $n<\left[L: M^{\infty}(S)\right]<\infty$. Since $L / M^{\infty}(S)$ is a finite separable extension, there exists a primitive element $f$ in $L$ with $L=\left[M^{\infty}(S)\right](f)$. By (2.3), $\left[L: M^{\infty}(S)\right] \leq n$, a contradiction. By the same reason we in fact have

$$
M^{\infty}(R)=\left[M^{\infty}(S)\right](f)
$$

for an $f$ in $M^{\infty}(R)$. Thus in particular to every $g \in M^{\infty}(R)$ there exist $n$ functions $q_{1}, \ldots, q_{n}$ in $M^{\infty}(S)$ such that

$$
g=q_{1} f^{n-1}+\cdots+q_{n-1} f+q_{n} .
$$

This last relation, however, will not be made use of in our reasoning in the sequel.

2.3. Fix a point $a$ in $S$ and consider the set

$$
A=\pi^{-1}(a)=\left\{a_{1}, \ldots, a_{n}\right\}
$$

in $R$. Here a point which is a branch point of order $m-1$ is appearing in $A$ as $m$ (identical) points in $\left\{a_{1}, \ldots, a_{n}\right\}$ and trivially every $f$ in $M^{\infty}(R)$ takes the same values at these points. But there can happen $f\left(a_{i}\right)=f\left(a_{j}\right)$ for every $f$ in $M^{\infty}(R)$ even for different two points $a_{i}$ and $a_{j}$. We say that $a_{i}$ and $a_{j}$ in $A$ are equivalent, $a_{i} \sim a_{j}$ in notation, if $f\left(a_{i}\right)=f\left(a_{j}\right)$ for every $f$ in $M^{\infty}(R)$ (weakly nonseparated in the terminology of [13]; we do not know whether we can replace $M^{\infty}(R)$ by $H^{\infty}(R)$ or not in the above). Let

$$
A=A_{1} \cup \cdots \cup A_{k}, \quad A_{i} \neq \varnothing, \quad A_{i} \cap A_{j}=\varnothing \quad(i \neq j)
$$

be the decomposition of $A$ into equivalence classes so that $a_{i}, a_{j} \in A_{p}$ imply that $a_{i} \sim a_{j}$ and $a_{i} \in A_{i}, a_{j} \in A_{j}(i \neq j)$ imply that $a_{i} \nsim a_{j}$. Every $f \in M^{\infty}(R)$ thus takes a constant value on each $A_{i}$.

We say that $f \in M^{\infty}(R)$ separates $A$ if $f(A) \subset \mathbf{C}$ and $f\left(A_{i}\right) \neq f\left(A_{j}\right)(i \neq j)$. Here and hereafter we also use $f\left(A_{i}\right)$ to mean the single value in the set $f\left(A_{i}\right)$ to save notations. To see the existence of an $f \in M^{\infty}(R)$ separating $A$ we take a $g$ in $M^{\infty}(R)$ depending on $i$ such that $g\left(A_{1}\right) \neq g\left(A_{i}\right)(i \neq 1)$. By adding a constant if necessary we may assume that $g(A) \subset C^{*} \cup\{\infty\}$ and by considering $1 / g$ if necessary we can assume that $g(A) \subset \mathbf{C}$. Let $g_{i}$ be obtained from $g$ by subtracting $g\left(A_{i}\right)$ and then by dividing by $g\left(A_{1}\right)-g\left(A_{i}\right)$ so that $g_{i}(A) \subset \mathbf{C}$ and $g_{i}\left(A_{1}\right)=1$ and $g_{i}\left(A_{i}\right)=0$ $(i \neq 1)$. Let $h_{1}=g_{1} \cdots g_{k}$. Then $h_{1}(A) \subset \mathbf{C}$ and $h_{1}\left(A_{1}\right)=1$ and $h_{1}\left(A_{i}\right)=0$ for every $i \neq 1$. Construct $h_{j}(j=2, \ldots, k)$ in the similar fashion so that $h_{j}(A) \subset \mathbf{C}$ 
$(j=1, \ldots, k)$ and $h_{j}\left(A_{i}\right)=\delta_{j i}$ (the Kronecker delta) $(i, j=1, \ldots, k)$. Then $f=\sum_{i=1}^{k} \alpha_{i} h_{i} \in M^{\infty}(R)$ is the required if $\left\{\alpha_{1}, \ldots, \alpha_{k}\right\} \subset \mathbf{C}$ are different by pairs.

2.4. First we prove that $R$ is stable under the assumption that $S$ is stable. Clearly $H^{\infty}(R) \neq \mathbf{C}$ because $H^{\infty}(R) \supset H^{\infty}(S) \neq \mathbf{C}$. Take an arbitrary valuation $v$ on $M^{\infty}(R)$. We have to show that $v$ is a point valuation on $M^{\infty}(R)$. Since $M^{\infty}(S) \subset M^{\infty}(R), v=v \mid M^{\infty}(S)$ is also a valuation on $M^{\infty}(S)$ if it is nontrivial. Contrariwise we assume that $v$ is trivial on $M^{\infty}(S)$, i.e. $v\left(M^{\infty}(S)\right)=\{0\}$. Choose an $f$ in $M^{\infty}(R)$ with $v(f)<0$. By $(2.2)$

$$
f^{n}=-\left(c_{1} f^{n-1}+\cdots+c_{n-1} f+c_{n}\right) .
$$

Since $v\left(c_{i} f^{n-i}\right)=(n-i) v(f)(i=1, \ldots, n),(1.1)$ implies that $n v(f) \geq(n-1) v(f)$ (cf. the argument in the proof of (i) below), a contradiction. Therefore $v$ is a valuation on $M^{\infty}(S)$ and by the stability of $S v$ is a point valuation on $M^{\infty}(S)$. On multiplying $v$ a suitable constant if necessary we can assume that $v(f)=\partial_{f}(a)$ $\left(f \in M^{\infty}(S)\right)$ for a fixed point $a$ in $S$.

Let $A=\pi^{-1}(a)=\left\{a_{1}, \ldots, a_{n}\right\}$ and $A=A_{1} \cup \cdots \cup A_{k}$ be the decomposition of $A$ as described in (2.6). We start by proving the following:

(i) $f(A) \subset \mathbf{C}^{*}$ implies that $v(f)=0$.

In fact, let $c_{1}, \ldots, c_{n}$ be $c$-functions associated with $f$. Since $f(A) \subset \mathbf{C}^{*}$, the relations (2.1) imply that $c_{1}, \ldots, c_{n}$ do not have any pole at $a$ and in particular $c_{n}(a) \neq 0$. Therefore

$$
v\left(c_{k}\right)=\partial_{c_{k}}(a) \geq 0 \quad(k=1, \ldots, n-1), \quad v\left(c_{n}\right)=\partial_{c_{n}}(a)=0 .
$$

In view of (2.2) with (1.1) and (1.2) we have

$$
\begin{aligned}
n v(f) & =v\left(f^{n}\right)=v\left(-f^{n}\right) \\
& =v\left(c_{1} f^{n-1}+\cdots+c_{n-1} f+c_{n}\right) \\
& \geq \min \left(v\left(c_{1}\right)+(n-1) v(f), \ldots, v\left(c_{n-1}\right)+v(f), v\left(c_{n}\right)\right) \\
& \geq \min ((n-1) v(f), \ldots, v(f), 0) \\
& =(n-j) v(f)
\end{aligned}
$$

for some $j=1, \ldots, n$. Thus we must conclude that $v(f) \geq 0$. Since we can apply the whole argument above to $1 / f$ because $(1 / f)(A) \subset \mathbf{C}^{*}$, we also conclude that $v(1 / f) \geq 0$ or $v(f) \leq 0$. Therefore we must have $v(f)=0$.

As the first consequence of (i) we have the following

(ii) $f(A) \subset \mathbf{C}$ implies that $v(f) \geq 0$.

In fact, $(f-\lambda)(A) \subset \mathbf{C}^{*}$ for a suitable $\lambda$ in $\mathbf{C}$ and hence $v(f-\lambda)=0$ by (i). Then $v(f)=v(f-\lambda+\lambda) \geq \min (v(f-\lambda), v(\lambda))=0$, as required.

Another consequence of (i) is the following

(iii) $v(f)>0$ implies $f\left(A_{i}\right)=0$ for some $i=1, \ldots, k$.

Contrariwise we assume that $f\left(A_{i}\right) \neq 0$ for every $i=1, \ldots, k$ so that $f(A) \subset$ $\mathbf{C}^{*} \cup\{\infty\}$. Then $(1 / f)(A) \subset \mathbf{C}$ and a fortiori by (ii) $v(1 / f) \geq 0$ or $v(f) \leq 0$, a contradiction.

2.5. We still continue to establish auxiliary results for $v$ which after all become trivial once $v$ turned out to be a point valuation. Next one is

(iv) $v(f) \geq 0$ implies the existence of a unique $\lambda$ in $\mathbf{C}$ such that $v(f-\lambda)>0$. 
This means that $v$ is distinguished. It is not at all known in general whether the distinguishedness of valuations on $M^{\infty}$ is a consequence or to be assumed, but in our present situation we can prove that it is certainly a consequence.

To see the uniqueness first suppose the existence of two such different $\lambda$ and $\lambda^{\prime}$ in $\mathbf{C}$. Then

$$
0=v\left(\lambda-\lambda^{\prime}\right)=v\left(\left(f-\lambda^{\prime}\right)-(f-\lambda)\right) \geq \min \left(v\left(f-\lambda^{\prime}\right), v(f-\lambda)\right)>0,
$$

a contradiction.

We next prove the existence of such a $\lambda$ in $\mathbf{C}$. If $f \equiv \lambda \in \mathbf{C}$, then $v(f-\lambda)=$ $v(0)=+\infty>0$. Thus we may assume that $f \in M^{\infty}(R) \backslash$ C. Consider a function $g=1 /(f-t)$. Then

$$
v(g)=-v(f-t) \leq-\min (v(f), v(t))=0
$$

for any $t$ in $\mathbf{C}$. By choosing a $t \in \mathbf{C}$ suitably we can assume that $g(A) \subset \mathbf{C}$ and by (ii) $v(g) \geq 0$. Hence $v(g)=0$ or equivalently $v(f-t)=0$ for some $t$ in $\mathbf{C}$. Then consider

$$
h=\left(g-g\left(a_{1}\right)\right) \cdots\left(g-g\left(a_{k}\right)\right) .
$$

Clearly $v(h) \geq 0$ along with $v(g)=0$. The $c$-functions $c_{j}$ of $h$ have zeros at $a$ $(j=1, \ldots, n)$ and hence $v\left(c_{j}\right)=\partial_{c_{j}}(a)>0$. Therefore we have as before

$$
\begin{aligned}
n v(h) & =v\left(h^{n}\right)=v\left(-h^{n}\right) \\
& =v\left(c_{1} h^{n-1}+\cdots+c_{n-1} h+c_{n}\right) \\
& \geq \min \left(v\left(c_{1} h^{n-1}\right), \ldots, v\left(c_{n-1} h\right), v\left(c_{n}\right)\right) \\
& =\min \left(v\left(c_{1}\right)+(n-1) v(h), \ldots, v\left(c_{n-1}\right)+v(h), v\left(c_{n}\right)\right) \\
& \geq \min \left(v\left(c_{1}\right), \ldots, v\left(c_{n}\right)\right)>0 .
\end{aligned}
$$

On the other hand, $0<v(h)=\sum_{j=1}^{k} v\left(g-g\left(a_{j}\right)\right)$ and a fortiori there exists a $j$ such that $v\left(g-g\left(a_{j}\right)\right)>0$. Since $v(g)=0$, we must have $g\left(a_{j}\right) \neq 0$ and therefore $f\left(a_{j}\right) \in$ C. Observe that

$$
\begin{aligned}
v\left(g-g\left(a_{j}\right)\right) & =v\left(1 /(f-t)-1 /\left(f\left(a_{j}\right)-t\right)\right) \\
& =v\left(\left(f\left(a_{j}\right)-f\right) /(f-t)\left(f\left(a_{j}\right)-t\right)\right) \\
& =v\left(f-f\left(a_{j}\right)\right)
\end{aligned}
$$

and thus $v(f-\lambda)>0$ if we choose $\lambda=f\left(a_{j}\right)$.

As a consequence of (iv) we can show a kind of converse of (iii) although the genuine converse of (iii) is not true:

(v) $f \mid A=0$ implies that $v(f)>0$.

In fact, by (ii), $v(f) \geq 0$. Then, by (iv), there exists a $\lambda \in \mathbf{C}$ such that $v(f-\lambda)>$ 0 . By (iii) there exists an $i=1, \ldots, k$ such that $(f-\lambda)\left(A_{i}\right)=0$. However $f \mid A=0$ shows that $\lambda=0$ and hence $v(f)>0$.

2.6. By the result in 2.3, we can find an $f_{0}$ in $M^{\infty}(R)$ which separates $A$. Since $f_{0}-\lambda$ also separates $A$ for every $\lambda \in \mathbf{C}$, we can assume, by (iv), that $v\left(f_{0}\right)>0$. By (iii) there is an $i=1, \ldots, k$ such that $f_{0}\left(A_{i}\right)=0$. By renumbering if necessary we may assume that $f_{0}\left(A_{1}\right)=0$. We have then the following sharpening of (i):

(vi) $f\left(A_{1}\right) \subset \mathbf{C}^{*}$ implies that $v(f)=0$. 
In fact, we take a positive number $\varepsilon$ sufficiently small, then $f+\varepsilon f_{0}$ never vanishes on $A$ but possibly takes the value $\infty$. Again taking a positive number $\eta$ small enough we see that $\left(1 /\left(f+\varepsilon f_{0}\right)+\eta f_{0}\right)(A) \subset \mathbf{C}^{*}$. By (i) we have

$$
0=v\left(1 /\left(f+\varepsilon f_{0}\right)+\eta f_{0}\right) \geq \min \left(v\left(1 /\left(f+\varepsilon f_{0}\right)\right), v\left(f_{0}\right)\right),
$$

and since $v\left(f_{0}\right)>0$, we must have $v\left(1 /\left(f+\varepsilon f_{0}\right)\right)=0$ or equivalently $v\left(f+\varepsilon f_{0}\right)=0$. Then, similarly as above,

$$
0=v\left(f+\varepsilon f_{0}\right) \geq \min \left(v(f), v\left(f_{0}\right)\right)
$$

implies that $v(f)=0$, as required.

We then have

(vii) $v(f)>0$ implies that $f\left(A_{1}\right)=0$.

There are three possibilities: $f\left(A_{1}\right)=0, f\left(A_{1}\right) \subset \mathbf{C}^{*}$, and $f\left(A_{1}\right)=\infty$. The second cannot be the case by (vi). If the third case happens, then $(1 / f)\left(A_{1}\right)=0$ and $(1 / f+1)\left(A_{1}\right) \subset \mathbf{C}^{*}$. Hence by (vi) $v(1 / f+1)=0$ and $v(1 / f)=v(1 / f+1-1) \geq$ $\min (v(1 / f+1), v(1))=0$ or $v(f) \leq 0$. Therefore we must have the first case.

2.7. We now come to the final step of the argument started in 2.4. Let $f_{1} \in$ $M^{\infty}(R)$ be such that

$$
v\left(f_{1}\right)=d=\min \left\{v(f): f \in M^{\infty}(R), v(f)>0\right\}>0 .
$$

Observe that $d$ divides every $v(f)\left(f \in M^{\infty}(R)\right)$. Let $p \in A_{1}$ be such that

$$
\partial_{f_{1}}(p)=e=\min \left\{\partial_{f_{1}}(q): q \in A_{1}\right\},
$$

which is positive by (vii). Take an arbitrary $f \in M^{\infty}(R)$ and let $\partial_{f}(p)=t$. Then

$$
\partial_{f^{e} / f_{1}^{t}}(p)=e \partial_{f}(p)-t \partial_{f_{1}}(p)=0
$$

and $\left(f^{e} / f_{1}^{t}\right)(p) \in \mathbf{C}^{*}$ so that $\left(f^{e} / f_{1}^{t}\right)\left(A_{1}\right) \subset \mathbf{C}^{*}$. By (vi) we see that $v\left(f^{e} / f_{1}^{t}\right)=0$ or $v(f)=(d / e) t=(d / e) \partial_{f}(p)$. Thus we have seen that $v$ is a point valuation on $M^{\infty}(R)$, i.e. $R$ is stable.

2.8. Finally we prove that $S$ is stable if $R$ is stable. For the purpose we take any valuation $v$ on $M^{\infty}(S)$. Recall the well-known fact about extensions of valuations (cf. e.g. [10, pp. 299-300]): Any discrete valuation on a field can be extended to a discrete valuation on its finite algebraic extension field.

Therefore, in view of (2.4) or (2.5), we may assume, on replacing $v$ by its equivalent if necessary, that $v$ is the restriction on $M^{\infty}(S)$ of a valuation $V$ on $M^{\infty}(R)$, i.e. $v=V \mid M^{\infty}(S)$. By the stability of $R$, there exists a point $b$ in $R$ and a constant $t$ such that $V(f)=t \partial_{f}(b)$ for every $f$ in $M^{\infty}(R)$. In particular we have

$$
v(f)=t \partial_{f \circ \pi}(b) \quad\left(f \in M^{\infty}(S)\right) .
$$

Let $a=\pi(b) \in S$ and $e$ be the multiplicity of $b$ over $a$. Then we have the relation

$$
\partial_{f \circ \pi}(b)=e \partial_{f}(a) \quad\left(f \in M^{\infty}(S)\right),
$$

and hence $v(f)=t e \partial_{f}(a)$ for every $f \in M^{\infty}(S)$, i.e. $v$ is a point valuation on $M^{\infty}(S)$ and thus $S$ is stable.

The proof of Theorem 1 is herewith complete. 


\section{Stable surfaces.}

3.1. It is surprising that not many stable surfaces are known. Nonstable surfaces are in plenty. Let $W$ be a surface such that $M^{\infty}(W)$ separates the points in $W$. An example of such a $W$ is a plane region with $W \notin O_{A B}$ and in particular a bounded plane region. Let $W_{0}$ be the surface obtained from the above surface $W$ by removing a point $a$ in $W$. Clearly any $f \in M^{\infty}\left(W_{0}\right)$ can be continued to the $f \in M^{\infty}(W)$ and $v(f)=\partial_{f}(a)$ can be defined. Then $v$ is a valuation on $M^{\infty}\left(W_{0}\right)$ but $v$ is seen not to be a point valuation on $M^{\infty}\left(W_{0}\right)$. If $M^{\infty}(W)$ does not separate the points in $W$, then $W_{0}$ can be stable (cf. Example 2 below). The above $W_{0}$ is a trivial example but a nontrivial nonstable plane region will be given in $\S 4$. The following is, in essence, the only one known stable surface (cf. Example 1 below):

THEOREM 2. The open unit disk $\Delta$ is stable.

Take an arbitrary valuation $v$ on $M^{\infty}(\Delta)$ and we have to show that $v$ is a point valuation on $M^{\infty}(\Delta)$. For the purpose we consider the identity function $I$ defined by $I(z) \equiv z$ on C. Since $I \in H^{\infty}(\Delta), v(I) \geq 0$ by (1.3). In 3.2, we will show that there exists an $a$ in $\Delta$ such that $v(I-a)>0$, which is an essential part of the proof of the above theorem. If the distinguishedness of $v$ is assumed here, then this is almost trivial. The proof is then completed in 3.3, which is rather a trivial part.

3.2. We will derive a contradiction from the erroneous assumption that $v(I-a)=$ 0 for every $a$ in $\Delta$.

Since $v$ is not trivial, there exists an $f$ in $H^{\infty}(\Delta)$ such that $v(f)>0$.

If $f$ has only a finite number of zeros $z_{1}, \ldots, z_{m}$ in $\Delta$, then $h=f /\left(I-z_{1}\right) \ldots$ $\left(I-z_{m}\right) \in H^{\infty}(\Delta)$ and hence $v(h)=v(f)>0$. Since $h \neq 0$ on $\Delta$, there exists the $n$th root $h^{1 / n}$ in $H^{\infty}(\Delta)$ for any $n \in \mathrm{N}$ and therefore $v(h)=n v\left(h^{1 / n}\right)$ implies that $v(h)=0$, a contradiction.

Therefore $f$ has infinitely many zeros in $\Delta$. We denote by $\left\{a_{n}\right\}_{n=1}^{\infty}$ the different zeros of $f$ and by $s_{n}$ the multiplicity of $a_{n}(n \in \mathbf{N})$. We indicate this by $\left\{a_{n}^{s_{n}}\right\}_{n=1}^{\infty}$. Let $B$ be the Blaschke product with $\left\{a_{n}^{s_{n}}\right\}_{n=1}^{\infty}$ its zero set:

$$
B(z)=\prod_{n=1}^{\infty}\left(\frac{-\bar{a}_{n}}{\left|a_{n}\right|}\right)^{s_{n}}\left(\frac{z-a_{n}}{1-\bar{a}_{n} z}\right)^{s_{n}} .
$$

Since $f / B \in H^{\infty}(\Delta)$ and has no zeros on $\Delta$, we see that $v(f / B)=0$ as above and a fortiori

$$
v(B)=v(f)>0 .
$$

By the Blaschke theorem, the zero set $\left\{a_{n}^{s_{n}}\right\}_{n=1}^{\infty}$ satisfies

$$
\sum_{n=1}^{\infty} s_{n}\left(1-\left|a_{n}\right|\right)<\infty
$$

We can find a nondecreasing sequence $\left\{t_{n}\right\}_{n=1}^{\infty}$ in $\mathbf{N}$ such that $\lim _{n \rightarrow \infty} t_{n}=\infty$ and yet

$$
\sum_{n=1}^{\infty} t_{n} s_{n}\left(1-\left|a_{n}\right|\right)<\infty
$$

Again by the Blaschke theorem there exists the Blaschke product $C$ with $\left\{a_{n}^{s_{n} t_{n}}\right\}_{n=1}^{\infty}$ its zero set. Similarly let $B_{m}$ and $C_{m}$ be the Blaschke products with $\left\{a_{n}^{s_{n}}\right\}_{n=m}^{\infty}$ and $\left\{a_{n}^{s_{n} t_{n}}\right\}_{n=m}^{\infty}$ their zero sets, respectively. 
Again by $v\left(I-a_{n}\right)=0(n=1,2, \ldots)$ and by the fact that $C_{m} / B_{m}^{t_{m}} \in H^{\infty}(\Delta)$ $(m=1,2, \ldots)$, we have

$$
v(C)=v\left(C_{m}\right) \geq v\left(B_{m}^{t_{m}}\right)=t_{m} v\left(B_{m}\right)=t_{m} v(B),
$$

i.e. $t_{m} \leq v(C) / v(B)<\infty(m=1,2, \ldots)$, contradicting the choice of $\left\{t_{m}\right\}$ :

$$
\lim _{m \rightarrow \infty} t_{m}=\infty
$$

3.3. We have seen that there exists an $a$ in $\Delta$ with the property that $e=$ $v(I-a)>0$. Let $g \in H^{\infty}(\Delta)$ and $g(a) \neq 0$. Since $(g-g(a)) /(I-a) \in H^{\infty}(\Delta)$, we have $v(g-g(a)) \geq v(I-a)>0$. Hence $g(a) \neq 0$ implies $v(g)=0$.

Let $f \in H^{\infty}(\Delta) \backslash \mathbf{C}$ and $\partial_{f-f(a)}(a)=t$. Then $(f-f(a)) /(I-a)^{t}$ belongs to $H^{\infty}(\Delta)$ and does not vanish at $a$. Hence by the above the value of $v$ at this function is zero so that $v(f-f(a))=e t$. In case $f(a)=0$ we then have $v(f)=e \partial_{f}(a)$. If $f(a) \neq 0$, then $v(f-f(a))=e t>0$ implies that $v(f)=0=e \partial_{f}(a)$. Thus in any case $v(f)=e \partial_{f}(a)$ for every $f \in H^{\infty}(\Delta)$ and therefore for every $f \in M^{\infty}(\Delta)$, i.e. $v$ is a point valuation.

The proof of Theorem 2 is herewith complete.

3.4. An $n$ sheeted disk $\Delta_{n}$ is an $n$ sheeted unlimited covering surface of the open unit disk $\Delta$. We also call $\Delta_{n}$ a finitely sheeted disk without specifying the sheet number $n$. The following is due to Frank Forelli [4] to whom the author is very grateful for many valuable suggestions and information:

EXAMPLE 1. Any finitely sheeted disk is stable.

The result follows immediately from Theorems 1 and 2. Plane regions bounded by finitely many mutually disjoint nondegenerate continua are finitely sheeted disks by the Bieberbach-Grunsky theorem (cf. e.g. [16]) or more generally finite open Riemann surfaces are finitely sheeted disks by the Ahlfors theorem [1]. Here a finite open Riemann surface is a surface obtained from a closed surface by removing a finite number of mutually disjoint nondegenerate continua. Hence as a special case of the above example we have

\section{COROLLARY. Finite open Riemann surfaces are stable.}

In an old paper [12] Royden proved that finite open Riemann surfaces are weakly stable. Thus the above Forelli result may be viewed as a generalization of the Royden result in two ways. First the weak stability is strengthened to the stability for finite open surfaces. Second there are not only finite surfaces but also surfaces of infinite genus among finitely sheeted disks.

3.5. We proceed to our second example of stable surfaces which cannot be represented as a finitely sheeted disk. Let $\Delta_{2}$ be a 2 sheeted disk $\left(\Delta_{2}, \Delta, \pi\right)$ with the sequence $\left\{x_{n}\right\}_{n=1}^{\infty}$ of projections $x_{n}$ of branch points in $\Delta_{2}$ lying over the positive real axis such that

$$
0<x_{1}<x_{2}<\cdots<x_{n}<\cdots<1, \quad \lim _{n \rightarrow \infty} x_{n}=1
$$

and moreover

$$
\sum_{n=1}^{\infty}\left(1-x_{n}\right)=+\infty
$$


Let $\left\{\sigma_{k}\right\}_{k=1}^{\infty}$ be a sequence of disjoint closed disks contained in $\Delta \cap\{\operatorname{Re} z<0\}$ converging to -1 . We denote by $E$ one of two connected pieces of

$$
\pi^{-1}(\Delta \cap\{\operatorname{Re} z<0\}) .
$$

Finally let

$$
U=\Delta_{2} \backslash E \cap \pi^{-1}\left(\bigcup_{k=1}^{\infty} \sigma_{k}\right) .
$$

EXAMPLE 2. The surface $U$ in (3.3) is of infinite genus, of infinite connectivity and not representable as a finitely sheeted disk but stable.

To use the Myrberg type argument take any $f \in H^{\infty}(U)$ and consider $h(z)=$ $\left(f\left(z_{1}\right)-f\left(z_{2}\right)\right)^{2}$ on $|z-1 / 2|<1 / 2$ where $\pi^{-1}(z)=\left\{z_{1}, z_{2}\right\}$. The Blaschke theorem with (3.2) implies that $h(z) \equiv 0$. This means that $f$ can be continued to $\Delta_{2}$ so that

$$
H^{\infty}(U)=H^{\infty}\left(\Delta_{2}\right)=H^{\infty}(\Delta) \circ \pi .
$$

As the first consequence of (3.4), there is no proper analytic map of $U$ onto $\Delta$ so that $U$ is not a finitely sheeted disk. The relation (3.4) also assures that $U$ is stable.

3.6. Although $U$ in Example 2 is stable and not a finitely sheeted disk, we are quite unsatisfied with $U$ because of condition (3.2) by which $U$ is made essentially a disk in the $H^{\infty}$ sense. In this connection the following is the most interesting and also important.

\section{OPEN PROBLEM 2. Is there any stable plane region of infinite connectivity?}

Probably one should try to determine whether the following concrete regions are stable or not:

(1) A region obtained from the punctured open unit disk $\Delta_{0}$ by removing a sequence of mutually disjoint closed disks with centers on the positive real axis in $\Delta_{0}$ contained in $\Delta_{0}$ that accumulates only at $z=0$ (a Zalcman $L$-domain [17]);

(2) A region obtained from the open unit disk $\Delta$ by removing a sequence of mutually disjoint closed disks with centers on the positive real axis in $\Delta$ contained in $\Delta$ that accumulates only at $z=1$;

(3) A Denjoy domain [6], i.e. a region obtained from the plane by removing a closed set on the real axis. Regions in (1) and (2) are conformally special cases of Denjoy domains.

In $\S 4$ we will see that there exists a nonstable region of type (1) and in the final $\S 5$ we will prove a theorem assuring that regions of type (2) are weakly stable. Thus the problem is still widely open.

\section{Maximality and stability.}

4.1. We say that a Riemann surface $W^{\prime}$ is an $H^{\infty}$-extension of a Riemann surface $W$ if $W$ is a subsurface of $W^{\prime}$ and $H^{\infty}\left(W^{\prime}\right) \mid W=H^{\infty}(W)$. A Riemann surface $W$ is said to be $H^{\infty}$-maximal if $W^{\prime}=W$ for any $H^{\infty}$-extension $W^{\prime}$ of $W$. We are interested in the relation between the $H^{\infty}$-stability and the $H^{\infty}$-maximality for Riemann surfaces and especially for plane regions. 
First of all how about the implication of the $H^{\infty}$-maximality from the $H^{\infty}$ stability? This is not true in general because the surface $U$ in Example 2 is $H^{\infty}$ stable but not $H^{\infty}$-maximal since $\Delta_{2}$ is the proper $H^{\infty}$-extension of $U$. However, as is easily seen, the $H^{\infty}$-stability always implies the $H^{\infty}$-maximality for plane regions. It would be very nice if the converse of this is true but unfortunately this is not the case. Thus the implication of the $H^{\infty}$-stability from the $H^{\infty}$-maximality is not true even for plane regions. We will give such an example in this section in the sequel.

4.2. We denote by $\Delta(c, r)(\bar{\Delta}(c, r)$, resp.) the open (closed, resp.) disk with the center $c$ and the radius $r$. Take a sequence $\left\{r_{k}\right\}_{1}^{\infty}$ of positive numbers $r_{k}$ such that

$$
2^{-k-1}+r_{k+1}<2^{-k}-r_{k} \quad(k=1,2, \ldots) .
$$

This simply means that $\left\{\sigma_{k}\right\}_{1}^{\infty}$ is the sequence of mutually disjoint closed disks $\sigma_{k}=$ $\bar{\Delta}\left(2^{-k}, r_{k}\right)(k=1,2, \ldots)$ contained in the punctured unit disk $\Delta_{0}=\Delta(0,1) \backslash\{0\}$. We then consider the plane region

$$
X=\Delta_{0} \backslash \bigcup_{k=1}^{\infty} \sigma_{k}
$$

which is an $L$-domain in the sense of Zalcman (cf. 3.6(1)). In addition to (4.1) we consider the condition

$$
\sum_{k=1}^{\infty} \frac{k}{\log \left(1 / r_{k}\right)}<\infty
$$

for $X$ (cf. [5, p. 74]), which is nothing but the necessary and sufficient condition for $z=0$ to be an irregular boundary point of $X$ in the sense of the potential theory (the Wiener criterion). Then we have the following.

EXAMPLE 3. The region $X$ in (4.2) is always $H^{\infty}$-maximal but, if the condition (4.3) is postulated, then $X$ is not $H^{\infty}$-stable (and actually $X$ is not even weakly $H^{\infty}$-stable).

The proof will be given in 4.3-4.8.

4.3. We first maintain that $X$ is always $H^{\infty}$-maximal. For the purpose we only have to show that there exists an $f_{\varsigma}$ in $H^{\infty}(X)$ for any $\varsigma \in \partial X$ such that $f_{\varsigma}$ has a singularity at $\zeta$, i.e. $f_{\zeta}$ cannot be holomorphically continued to a neighborhood of 5 .

In case $|\zeta|=1$ the restriction $f_{\varsigma}$ to $X$ of a Blaschke product on $\Delta=\Delta(0,1)$ whose zeros accumulate at $\zeta$ is a required since otherwise we must have $f_{\zeta} \equiv 0$. In case $\varsigma \in \partial \sigma_{k}(k=1,2, \ldots)$ we map the region $\left\{r_{k}<\left|z-2^{-k}\right| \leq \infty\right\}$ in the extended plane conformally onto the unit disk $\Delta$ by the Riemann mapping function $\varphi$ with $\varphi(\varsigma)=1$ and next take a Blaschke product $B$ on $\Delta$ whose zeros accumulate at 1 . The restriction $f_{\varsigma}$ of $B \circ \varphi$ to $X$ is, as above, a required.

Finally in case $\varsigma=0$ we take a sequence $\left\{\varepsilon_{k}\right\}_{1}^{\infty}$ of positive numbers $\varepsilon_{k}$ with $\sum_{k=1}^{\infty} \varepsilon_{k} / r_{k}<\infty$. The function $f_{0}$ defined by

$$
f_{0}(z)=\sum_{k=1}^{\infty} \frac{\varepsilon_{k}}{z-2^{-k}} \quad(z \in X)
$$


belongs to $H^{\infty}(X)$. Actually $f_{0}$ can be continued meromorphically to $\Delta_{0}$ and its poles cluster to $\zeta=0$. Thus $f_{0}$ cannot be holomorphically continued to $\zeta=0$.

4.4. We now assume the condition (4.3) for $X$ which is seen to be equivalent to the existence of a sequence $\{n(k)\}_{k=1}^{\infty}$ of positive numbers $n(k)$ such that

$$
r_{k}=2^{-n(k) k} \quad(k=1,2, \ldots), \quad \sum_{k=1}^{\infty} \frac{1}{n(k)}<\infty .
$$

Needless to say we have $n(k) \rightarrow \infty$ as $k \rightarrow \infty$.

We take another sequence of closed disks $\tau_{k}=\bar{\Delta}\left(2^{-k}, \sqrt{r_{k}}\right)(k=1,2, \ldots)$. These disks are contained in $\Delta_{0}$ and mutually disjoint for sufficiently large $k$. By changing the radii of $\tau_{k}$ suitably, if necessary, for the first finite number of disks $\tau_{k}$ under the restrictions $\tau_{k} \supset \sigma_{k}$, we may assume that $\tau_{k}$ are all contained in $\Delta_{0}$ and mutually disjoint. Then consider an auxiliary region

$$
Y=\Delta_{0} \backslash \bigcup_{k=1}^{\infty} \tau_{k}
$$

which is a subregion of $X$.

4.5. For later use in 4.7 here we remark the existence of a strictly positive harmonic function $p(z)$ on $Y$ with boundary values zero on $\partial Y \backslash\{0\}$ such that

$$
p(z) \leq \log (1 /|z|)
$$

for every $z$ in $Y$.

To see this first observe that condition (4.3) is equivalent to $\sum_{k=1}^{\infty} k / \log \left(1 / \sqrt{r_{k}}\right)$ $<\infty$. Therefore the point $z=0$ is also an irregular boundary point for the smaller region $Y$ in the sense of the harmonic Dirichlet problem. Let $p(z, \varsigma)$ be the harmonic Green's function for the region $Y$. The Bouligand theorem (cf. e.g. [16]) says that

$$
\lim _{\zeta \in Y} \sup _{\zeta \rightarrow 0} p(z, \varsigma)>0
$$

for one and hence for every $z$ in $R$. By the Harnack principle, there exists a sequence $\left\{\zeta_{n}\right\}$ in $Y$ convergent to 0 such that $\left\{p\left(z, \zeta_{n}\right)\right\}$ converges to a strictly positive harmonic function $p(z)$ on $Y$ uniformly on every compact subset of $Y$. Since each $p\left(z, \zeta_{n}\right)$ has vanishing boundary values on $\partial Y \backslash\{0\}$ and each component of $\partial Y \backslash\{0\}$ is a circle, the limit function $p(z)$ must have boundary values zero on $\partial Y \backslash\{0\}$. Moreover observe that

$$
p\left(z, \zeta_{n}\right)<\log \left|\frac{1-\bar{\zeta}_{n} z}{z-\varsigma_{n}}\right|
$$

on $Y$. On making $n \rightarrow \infty$ we obtain (4.5).

4.6. We denote by $\gamma_{k}$ the circle $\partial \sigma_{k}$ with negative direction $(k=1,2, \ldots)$ and by $\gamma_{0}$ the circle $\partial \Delta$ with positive direction. Any function $f \in H^{\infty}(X)$ has angular boundary values almost everywhere on each $\gamma_{k}$ defining a function in $L^{\infty}\left(\gamma_{k}\right)$ which we also denote by $f(k=0,1, \ldots)$. In view of $(4.4)$ the Cauchy formula implies that

$$
f^{(n)}(z)=\sum_{k=0}^{\infty} \frac{n !}{2 \pi i} \int_{\gamma_{k}} \frac{f(\varsigma)}{(\varsigma-z)^{n+1}} d \varsigma \quad(n=0,1, \ldots)
$$


for every $z$ in $X$ for any $f$ in $H^{\infty}(X)$, where $f^{(n)}$ is the $n$th derivative with the convention $f^{(0)}=f$.

Although $f(0)$ and $f^{(n)}(0)(n=1,2, \ldots)$ cannot be defined in their genuine senses for $f \in H^{\infty}(X)$ in general unless $f$ is holomorphically continued to $z=0$, we can define a formal $n$th derivative $f^{(n)}(0)$ of $f$ at $z=0$ with the convention $f^{(0)}(0)=f(0)$ by

$$
f^{(n)}(0)=\sum_{k=0}^{\infty} \frac{n !}{2 \pi i} \int_{\gamma_{k}} \frac{f(\varsigma)}{\varsigma^{n+1}} d \zeta \quad(n=0,1, \ldots)
$$

whose existence is assured again by (4.4). Of course $f^{(n)}$ coincides with the genuine $n$th derivative of $f$ at $z=0$ if $f$ is holomorphically extended to $z=0(n=0,1, \ldots)$.

Consider (4.6) for $z \in \bar{Y} \backslash\{0\}$. In view of (4.4) we see that

$$
\max _{\zeta \in \gamma_{k}}\left|\frac{f(\varsigma)}{(\zeta-z)^{n+1}}\right| \leq \frac{\|f\|_{X}}{\left(\sqrt{r_{k}}-r_{k}\right)^{n+1}} \leq \frac{2^{-k}}{r_{k}}
$$

for sufficiently large $k$. Thus the absolute value of the integrand in the integral of (4.6) for $z \in \bar{Y} \backslash\{0\}$ is dominated by an integrable step function independent of $z$. Therefore the Lebesgue dominated convergence theorem allows us to take the limit as $z \rightarrow 0$ in (4.6) under the integral sign to conclude

$$
f^{(n)}(0)=\lim _{z \in \bar{Y} \backslash\{0\}, z \rightarrow 0} f^{(n)}(z) \quad(n=0,1, \ldots) .
$$

From (4.8) it follows that the mapping $f \mapsto f^{(n)}(0)$ is a linear functional on $H^{\infty}(X)$,

$$
(\alpha f+\beta g)^{(n)}(0)=\alpha f^{(n)}(0)+\beta g^{(n)}(0)
$$

for $f, g \in H^{\infty}(X)$ and $\alpha, \beta \in \mathbf{C}$ as the limiting case of the linearity of $f \mapsto f^{(n)}(z)$. Similarly, as the limiting case of the Leibniz formula at $z$ as $z \rightarrow 0$, we deduce the Leibniz formula at 0 :

$$
(f g)^{(n)}(0)=\sum_{j=0}^{n}\left(\begin{array}{c}
n \\
j
\end{array}\right) f^{(n-j)}(0) g^{(j)}(0)
$$

for $f, g \in H^{\infty}(X)$.

4.7. The meaning of (4.9) and (4.10) is that the mapping of the algebra $H^{\infty}(X)$ into the algebra of formal power series at $z=0$ given by

$$
f \mapsto \sum_{n=0}^{\infty} \frac{f^{(n)}(0)}{n !} z^{n}
$$

is an algebraic homomorphism. We now see that it is actually an into algebraic isomorphism, i.e. (4.11) is injective. In other words $f^{(n)}(0)=0(n=0,1, \ldots)$ for any $f$ in $H^{\infty}(X)$ implies that $f \equiv 0$ on $X$.

Contrary to the assertion assume that $f \not \equiv 0$ on $X$. By multiplying a nonzero constant if necessary we may assume that $|f| \leq 1$ on $\bar{Y} \backslash\{0\}$.

The following representation of $f$ with $f^{(n)}(0)=0(n=0,1, \ldots)$ plays a crucial role in our reasoning:

$$
f(z)=z^{n} \sum_{k=0}^{\infty} \frac{1}{2 \pi i} \int_{\gamma_{k}} \frac{f(\zeta)}{\zeta^{n}(\zeta-z)} d \zeta \quad(n=0,1, \ldots) .
$$


We show this by the mathematical induction on $n$. This is certainly true for $n=0$ since it is nothing but (4.6) with $n=0$. Assume (4.12) for $n=m$. Then, by (4.7) with $n=m$ and $f^{(m)}(0)=0$, we proceed as follows:

$$
\begin{aligned}
f(z) & =f(z)-z^{m} f^{(m)}(0) \\
& =z^{m} \sum_{k=0}^{\infty} \frac{1}{2 \pi i} \int_{\gamma_{k}} f(\varsigma)\left(\frac{1}{\varsigma^{m}(\varsigma-z)}-\frac{1}{\varsigma^{m+1}}\right) d \zeta \\
& =z^{m+1} \sum_{k=0}^{\infty} \frac{1}{2 \pi i} \int_{\gamma_{k}} \frac{f(\varsigma)}{\varsigma^{m+1}(\varsigma-z)} d \varsigma
\end{aligned}
$$

which shows that (4.12) for $n=m+1$ holds, and the induction is herewith complete.

We denote by $C_{n}$ the supremum of $\left|f(z) / z^{n}\right|$ when $z$ runs over $Y(n=0,1, \ldots)$. Then by (4.12) we see that

$$
\begin{aligned}
C_{n}= & \sup _{z \in Y}\left|\sum_{k=0}^{\infty} \frac{1}{2 \pi i} \int_{\gamma_{k}} \frac{f(\zeta)}{\zeta^{n}(\zeta-z)} d \zeta\right| \\
& \leq \sum_{k=0}^{\infty} \frac{1}{2 \pi} \int_{\gamma_{k}} \frac{1}{|\zeta|^{n}\left(\sqrt{r_{k}}-r_{k}\right)}|d \zeta|<\infty
\end{aligned}
$$

in view of (4.4). Hence by (4.12) we have $|f(z)| \leq C_{n}|z|^{n}$ for any $z$ in $\bar{Y} \backslash\{0\}$ or equivalently $\log (1 /|f(z)|)$ dominates the function $n \log (1 /|z|)-\log C_{n}$ on $\bar{Y} \backslash\{0\}$ for every $n=0,1, \ldots$ Thus in particular by (4.5) we obtain the following

$$
\log \frac{1}{|f(z)|} \geq n p(z)-\log C_{n} \quad(n=0,1, \ldots)
$$

for every $z$ in $\bar{Y} \backslash\{0\}$. Consider a superharmonic function

$$
s(z)=s_{n, \varepsilon}(z)=\log \frac{1}{|f(z)|}+\varepsilon \log \frac{1}{|z|}-n p(z)
$$

on $\bar{Y} \backslash\{0\}$ for an arbitrarily fixed positive number $\varepsilon$. Since $|f| \leq 1$ on $\bar{Y}$ and $p=0$ on $\partial Y \backslash\{0\}$, we see that the boundary values of $s$ on $\partial Y \backslash\{0\}$ are nonnegative. By (4.13) and the effect of the term $\varepsilon \log (1 /|z|)$ we have $\lim _{z \in Y, z \rightarrow 0} s(z)=\infty$. Therefore we can conclude that

$$
\lim _{z \in Y} \inf _{z \rightarrow \varsigma} s(z) \geq 0 \quad(\varsigma \in \partial Y) .
$$

By the minimum principle for superharmonic functions we see that $s(z)=s_{n, \varepsilon}(z) \geq$ 0 for every $z$ in $Y$. On letting $\varepsilon$ tend to zero we finally conclude that

$$
\log (1 /|f(z)|) \geq n p(z) \quad(n=0,1, \ldots)
$$

for any $z$ in $Y$, which is a contradiction.

4.8. We now define a valuation $v$ on $M^{\infty}(X)$ which is not a point valuation on $M^{\infty}(X)$ so that $X$ is not stable. We will also show that $v$ is distinguished so that $X$ is not even weakly stable.

First for each $f$ in $H^{\infty}(X) \backslash\{0\}$ we set

$$
v(f)=\min \left\{n: f^{(n)}(0) \neq 0\right\}
$$


which is seen by 4.7 to be a nonnegative integer. We set $v(0)=+\infty$. By (4.10) we see that

$$
v(f g)=v(f)+v(g) \quad\left(f, g \in H^{\infty}(X)\right)
$$

and by (4.9) with $\alpha=\beta=1$ we deduce

$$
v(f+g) \geq \min (v(f), v(g)) \quad\left(f, g \in H^{\infty}(X)\right) .
$$

For any pair of functions $f_{1}$ and $f_{2}$ in $H^{\infty}(X)$ with $f_{2} \not \equiv 0$ we set

$$
v\left(f_{1} / f_{2}\right)=v\left(f_{1}\right)-v\left(f_{2}\right) \text {. }
$$

The value $v\left(f_{1} / f_{2}\right)$ is certainly determined uniquely by the ratio, i.e. $f_{1} / f_{2}=f_{3} / f_{4}$ implies $v\left(f_{1} / f_{2}\right)=v\left(f_{3} / f_{4}\right)$. In fact, applying (4.14) to $f_{1} f_{4}=f_{2} f_{3}$ we obtain $v\left(f_{1}\right)-v\left(f_{2}\right)=v\left(f_{3}\right)-v\left(f_{4}\right)$ which means that $v\left(f_{1} / f_{2}\right)=v\left(f_{3} / f_{4}\right)$. Thus $v$ can be defined on $M^{\infty}(X)$.

Again by (4.14) we see that

$$
\begin{aligned}
v\left(\frac{f_{1}}{f_{2}} \cdot \frac{f_{3}}{f_{4}}\right) & =v\left(\frac{f_{1} f_{3}}{f_{2} f_{4}}\right)=v\left(f_{1} f_{3}\right)-v\left(f_{2} f_{4}\right) \\
& =\left(v\left(f_{1}\right)+v\left(f_{3}\right)\right)-\left(v\left(f_{2}\right)+v\left(f_{4}\right)\right) \\
& =\left(v\left(f_{1}\right)-v\left(f_{2}\right)\right)+\left(v\left(f_{3}\right)-v\left(f_{4}\right)\right) \\
& =v\left(\frac{f_{1}}{f_{2}}\right)+v\left(\frac{f_{3}}{f_{4}}\right)
\end{aligned}
$$

for $f_{1}, f_{2}, f_{3}, f_{4} \in H^{\infty}(X)$. Therefore $v$ is a group homomorphism of the multiplicative group $M^{\infty}(X)^{*}$ into the additive group $\mathbf{Z}$.

By using (4.15) we proceed as follows:

$$
\begin{aligned}
v\left(\frac{f_{1}}{g_{1}}+\frac{f_{2}}{g_{2}}\right) & =v\left(\frac{f_{1} g_{2}+f_{2} g_{1}}{g_{1} g_{2}}\right)=v\left(f_{1} g_{2}+f_{2} g_{1}\right)-v\left(g_{1} g_{2}\right) \\
& \geq \min \left(v\left(f_{1} g_{2}\right), v\left(f_{2} g_{1}\right)\right)-v\left(g_{1} g_{2}\right) \\
& =\min \left(v\left(f_{1}\right)+v\left(g_{2}\right), v\left(f_{2}\right)+v\left(g_{1}\right)\right)-\left(v\left(g_{1}\right)+v\left(g_{2}\right)\right) \\
& =\min \left(v\left(f_{1}\right)-v\left(g_{1}\right), v\left(f_{2}\right)-v\left(g_{2}\right)\right) \\
& =\min \left(v\left(\frac{f_{1}}{g_{1}}\right), v\left(\frac{f_{2}}{g_{2}}\right)\right)
\end{aligned}
$$

for $f_{1}, g_{1}, f_{2}, g_{2} \in H^{\infty}(X)$ so that $v$ is a valuation on $M^{\infty}(X)$.

We now show that $v$ is distinguished. Suppose $v(f / g) \geq 0\left(f, g \in H^{\infty}(X), g \not \equiv\right)$ and we have to find a $\lambda \in \mathbf{C}$ with $v(f / g-\lambda)>0$. If $v(f / g)>0$, then $\lambda=0$ is the required. Thus we may suppose $v(f / g)=0$ so that $v(f)=v(g)=n$ is a nonnegative integer, $f^{(n)}(0), g^{(n)}(0) \neq 0$, and

$$
\left(g^{(n)}(0) f-f^{(n)}(0) g\right)^{(m)}(0)=g^{(n)}(0) f^{(m)}(0)-f^{(n)}(0) g^{(m)}(0)=0
$$

for nonnegative integers $m<n$ if $n>0$ since $v(f)=v(g)=n$ and also for $m=n$. Therefore we have

$$
v\left(g^{(n)}(0) f-f^{(n)}(0) g\right)>n .
$$

Now taking $\lambda=f^{(n)}(0) / g^{(n)}(0)$ we see by $v\left(g^{(n)}(0) g\right)=v(g)=n$ that

$$
\begin{aligned}
v(f / g-\lambda) & =v\left(\left(g^{(n)}(0) f-f^{(n)}(0) g\right) /\left(g^{(n)}(0) g\right)\right) \\
& =v\left(g^{(n)}(0) f-f^{(n)}(0) g\right)-v\left(g^{(n)}(0) g\right)>0 .
\end{aligned}
$$


We take the identity function $I$ in $H^{\infty}(X)$, i.e. $I(z) \equiv z$. Then $v(I)=1$ since $I(0)=0$ and $I^{(1)}(0)=1$. However $\partial_{I}(a)=0$ for every $a \in X$ and therefore $v$ is not a point valuation on $M^{\infty}(X)$.

\section{Weak stability.}

5.1. Recall that a valuation $v$ on $M^{\infty}(W)$ is distinguished if $v(f) \geq 0$ for an $f \in M^{\infty}(W)$ implies the existence of a $\lambda \in \mathbf{C}$ such that $v(f-\lambda)>0$. The important point here is that $\lambda$ is uniquely determined by $f$. A Riemann surface $W$ is stable (weakly stable, resp.), or more formally $H^{\infty}$-stable (weakly $H^{\infty}$-stable, resp.), if $M^{\infty}(W)$ is nontrivial (i.e. $M^{\infty}(W) \neq \mathbf{C}$ ) and any valuation (distinguished valuation, resp.) on $M^{\infty}(W)$ is a point valuation on $M^{\infty}(W)$. Hence clearly we have the following inclusion relation:

\section{\{stable surfaces $\} \subset\{$ weakly stable surfaces $\}$.}

To determine whether the above inclusion is proper or not is both interesting and important, and the question is closely related to the Open Problem 1 stated in 1.3. Here we state the question as

OPEN PROBLEM 3. Does the weak stability automatically imply the stability for Riemann surfaces and especially for plane regions?

In $\S 4$ we have seen the existence of weakly nonstable (and of course nonstable) bounded plane region $X$ nontrivial in the sense that it is $H^{\infty}$-maximal and of infinite connectivity (Example 3). The purpose of this section is to show the existence of weakly stable bounded plane region $V$ of infinite connectivity. All stable plane regions we know are of finite connectivity (cf. 3.4 and Open Problem 2). Thus to examine whether the above $V$ is stable or not would be the very first step to resolve Open Problem 3 (and also 2) but even this seems to be tough enough.

5.2. In this section we consider mainly bounded plane regions $S$. Then the field $M^{\infty}(S)$ is nontrivial and the identity function $I$ belongs to $H^{\infty}(S)$ where $I(z) \equiv z$ $(z \in \mathbf{C})$. Let $v$ be any distinguished valuation on $M^{\infty}(S)$. By property (1.3) the value of $v$ at $I$ is nonnegative. Hence by the distinguishedness of $v$ there exists a unique point $a_{v}$ in $\mathbf{C}$ such that $v\left(I-a_{v}\right)>0$. For convenience we call such an $a_{v}$ the support of $v$. In order to be able to define the support $a_{v}$ of a valuation $v$ on $M^{\infty}(S)$ we are not using the full power of the distinguishedness of $v$ but only a part of it: There is an $a \in \mathbf{C}$ with $v(I-a)>0$ only for the function $I$. From this point of view the whole discussions in the sequel in this section can be applied to slightly more general valuations than distinguished ones but to avoid a possibly inessential complication we do not go into this matter any further.

We now introduce an entirely new notion of $H^{\infty}$-barrier. A boundary point $\zeta$ of a bounded plane region $S$ is said to have an $H^{\infty}$-barrier $b_{\varsigma}$ on $S$ if $(I-\varsigma)^{-n} \cdot b_{\varsigma}$ belongs to $H^{\infty}(S) \backslash\{0\}$ for every $n=0,1, \ldots$. We also say that $b_{\varsigma}$ is an $H^{\infty}$-barrier at $\varsigma$ on $S$. Thus an $H^{\infty}$-barrier $b_{\varsigma}$ on $S$ at $\varsigma \in \partial S$ is a nonzero bounded holomorphic function on $S$ vanishing at $\zeta$ from the inside of $S$ faster than any power $(I-\varsigma)^{n}$ $(n=1,2, \ldots)$.

Concerning properties of supports of valuations and also important roles played by $H^{\infty}$-barriers we have the following.

PROPOSITION 1. Let $v$ be a distinguished valuation on the field $M^{\infty}(S)$ over a bounded plane region $S$ and $a_{v}$ the support of $v$. Then $a_{v}$ belongs to the closure $\bar{S}$. 
Moreover $a_{v}$ belongs to $S$ if and only if $v$ is a point valuation at $a_{v}$. In addition a boundary point $\zeta$ of $S$ cannot be the support $a_{v}$ of any $v$ if $\varsigma$ has an $H^{\infty}$-barrier $b_{\varsigma}$.

Suppose first erroneously that $a_{v} \notin \bar{S}$. Then the function $1 /\left(I-a_{v}\right)$ belongs to $H^{\infty}(S)$ and its $v$-value must be nonnegative, or $v\left(I-a_{v}\right) \leq 0$, a contradiction.

Next suppose that $v$ is a point valuation at $b \in S$, i.e. $v=c \partial$. $(b)(c>0)$. Then $v(I-b)=c \partial_{I-b}(b)=c>0$ shows that $a_{v}=b \in S$. Conversely suppose $a=a_{v} \in S$. Then the whole discussions in 3.3 can be easily modified to a proof for $v$ to be a point valuation at $a_{v}$.

Finally suppose $\varsigma \in \partial S$ has an $H^{\infty}$-barrier $b_{\varsigma}$ on $S$ and contrariwise suppose that there exists a distinguished valuation $v$ on $M^{\infty}(S)$ such that its support $a_{v}=\varsigma$. Since $(I-\varsigma)^{-n} \cdot b_{\varsigma}$ belongs to $H^{\infty}(S)$, its $v$-value must be nonnegative or $v\left(b_{\varsigma}\right) \geq$ $n v\left(I-a_{v}\right)$ for every $n=1,2, \ldots$, contradicting $v\left(I-a_{v}\right)>0$.

COROLLARY. If every boundary point of a bounded plane region $S$ has an $H^{\infty}$. barrier on $S$, then $S$ is weakly stable.

5.3. In view of the corollary to Proposition 1 it is important to determine when a boundary point $\zeta$ of $S$ has an $H^{\infty}$-barrier. We start by giving a necessary condition:

PROPOSITION 2. If a boundary point $\varsigma$ of a bounded plane region $S$ has an $H^{\infty}$ barrier on $S$, then $\zeta$ is a regular boundary point of $S$ in the sense of the potential theory.

Contrariwise we assume that $\zeta$ is an irregular boundary point of $S$ in the sense of the potential theory. By applying a suitable Möbius transformation we may assume that $\zeta=0$ and $S \subset \Delta(0,1)$. By modifying the discussion in 4.5 we can find a strictly positive harmonic function $p(z)$ on $S$ with locally bounded boundary values on $\partial S \backslash\{0\}$ which are zero on the subset of $\partial S \backslash\{0\}$ consisting of regular boundary points of $S$ such that

$$
p \leq \log (1 /|I|) .
$$

Let $b_{0}$ be an $H^{\infty}$-barrier on $S$ at $\varsigma=0$. We can assume that $\left|b_{0}\right| \leq 1$ on $S$. Since $I^{-n} \cdot b_{0} \in H^{\infty}(S)$ for every $n=1,2, \ldots$, we have

$$
c_{n}=\sup _{S}\left|I^{-n} \cdot b_{0}\right|<\infty \quad(n=1,2, \ldots) .
$$

We now repeat the discussion in the last part of 4.7 with a suitable modification. First, from $\left|b_{0}\right| \leq c_{n}|I|^{n}$ it follows that $\log \left(1 /\left|b_{0}\right|\right)$ dominates $n \log (1 /|I|)-\log c_{n}$, and by (5.1), we have

$$
\log \left(1 /\left|b_{0}\right|\right) \geq n p-\log c_{n} .
$$

Choose a finite positive superharmonic function $q$ on $S$ with the infinite boundary values on the subset of $\partial S$ consisting of irregular boundary points of $S$. Consider a superharmonic function

$$
s(z)=s_{n, \varepsilon}(z)=\log \left(1 /\left|b_{0}\right|\right)+\varepsilon q-n p
$$

on $S$ for an arbitrary fixed positive number $\varepsilon$. By the properties of $p$ and $q$ we see that the boundary values of $s$ on $\partial S \backslash\{0\}$ are nonnegative. By (5.2) and again 
by the effect of the term $\varepsilon q$ at 0 we have $\lim _{z \in S, z \rightarrow 0} s(z)=\infty$. Therefore we can conclude that

$$
\lim _{z \in S} \inf _{z \rightarrow \varsigma} s(z) \geq 0 \quad(\varsigma \in \partial S) .
$$

Then, by the same fashion as in the last part of 4.7 , we conclude that

$$
\log \left(1 /\left|b_{0}\right|\right) \geq n p \quad(n=1,2, \ldots)
$$

on $S$, which is a contradiction since $b_{0} \not \equiv 0$ and $p>0$.

The proof of Proposition 2 is herewith complete.

It would be very nice if the converse of the above proposition is true but we have no idea at present and hence we state here the following problem.

OPEN PROBLEM 4. Does a boundary point $\zeta$ of a bounded plane region $S$ which is regular in the sense of the potential theory have an $H^{\infty}$-barrier on $S$ ?

Take the region $X$ in Example 3. If (4.3) is postulated, then the boundary point $\zeta=0$ is irregular and hence $\zeta=0$ does not have any $H^{\infty}$-barrier on $X$. We ask whether $\varsigma=0$ have an $H^{\infty}$-barrier or not if $\sum_{k=1}^{\infty} k / \log \left(1 / r_{k}\right)=\infty$, i.e. if $\varsigma=0$ is regular. This must be the first step to attack the above problem (cf. also the last paragraph of 5.6 below).

5.4. The following result is rather trivial but will be essentially made use of in the proof of Theorem 3 below which is the main result of this section. It is also in the positive direction to Problem 4.

PROPOSITION 3. Let $F$ be a closed subset of the open unit disk $\Delta$ such that $S=\Delta \backslash F$ is connected. Then any boundary point $\varsigma$ of $S$ lying on the unit circle $\partial \Delta$ has an $H^{\infty}$-barrier on $S$.

Suppose there exists an $H^{\infty}$-barrier $b_{\varsigma}$ on $\Delta$ at $\varsigma \in \partial \Delta$ with $\varsigma \in \partial S$. Then $b_{\varsigma} \mid S$ is an $H^{\infty}$-barrier on $S$ at $\varsigma$. Therefore we only have to find an $H^{\infty}$-barrier $b_{\varsigma}$ on $\Delta$ at $\varsigma \in \partial \Delta$.

Let $T=-\varsigma^{-1} \Delta+1$ which is in the right half plane $P=\{\operatorname{Re} w>0\}$. The point $w=0$ is in the boundary $\partial T$ which is the image of $\zeta$ under the mapping

$$
z \mapsto w=\tau(z)=-\varsigma^{-1}(z-\varsigma)
$$

from $\Delta$ onto $T$. Take the branch of $\sqrt{w}$ in $P$ with $\sqrt{1}=1$ and then consider

$$
b(w)=\exp (-1 / \sqrt{w})
$$

on $P$. Clearly $b \in H^{\infty}(P) \subset H^{\infty}(T)$ and $w^{-n} b(w)$ is bounded on $P$ for every $n \in \mathbf{N}$ since we can easily see that

$$
\lim _{w \in P, w \rightarrow 0} w^{-n} \cdot b(w)=0
$$

for every $n \in \mathrm{N}$. The required $b_{\zeta}$ is then given by

$$
b_{\varsigma}(z)=b(\tau(z))=b\left(-\varsigma^{-1}(z-\varsigma)\right) \text {. }
$$

It certainly belongs to $H^{\infty}(\Delta)$ and $(I-\varsigma)^{-n} \cdot b_{\varsigma}$ is bounded on $\Delta$ for every $n \in \mathbf{N}$ since, by (5.3), we have

$$
\begin{aligned}
\lim _{z \in \Delta, z \rightarrow \varsigma}(z-\varsigma)^{-n} b_{\varsigma}(z) & =\lim _{z \in \Delta, z \rightarrow \varsigma}\left(\frac{\tau(z)-\tau(\varsigma)}{z-\varsigma}\right)^{n} \tau(z)^{-n} b(\tau(z)) \\
& =\tau^{\prime}(\varsigma)^{n} \lim _{w \in T, w \rightarrow 0} w^{-n} b(w)=0 .
\end{aligned}
$$

Thus we have seen that $b_{\varsigma}$ is a required $H^{\infty}$-barrier on $\Delta$ at $\varsigma$. 
5.5. We now state our main result of this section. The region we consider here is a subregion of the extended plane and thus may not be bounded although it is conformally equivalent to a bounded plane region. At this point we remind the reader that the stability and weak stability are conformally invariant properties.

THEOREM 3. A region in the extended plane which is conformally equivalent to a region such that any connected component of its boundary is a nondegenerate continuum is weakly stable.

We denote by $R$ the region in question and we may suppose that $R$ is bounded and each connected component of the boundary $\partial R$ of $R$ is a nondegenerate continuum. Take any distinguished valuation $v$ on $M^{\infty}(R)$ and we have to show that $v$ is a point valuation on $M^{\infty}(R)$.

Let $a=a_{v}$ be the support of $v$ so that $a \in \bar{R}$ by Proposition 1. Since $v$ is a point valuation on $M^{\infty}(R)$ if and only if $a \in R$ again by Proposition 1, we will only have to derive a contradiction from the erroneous assumption that $a \in \partial R$.

Denote by $K$ the connected component of $\partial R$ that contains $a$ and $T$ the component of the complement of $K$ with respect to the extended plane which contains $R$. Then $T$ is a simply connected region in the extended plane whose boundary is $K$ that is a nondegenerate continuum. Hence $T$ can be mapped conformally onto the unit disk $\Delta$ by a Riemann mapping function $\varphi$. Observe that $F=\Delta \backslash \varphi(R)$ is a closed subset of $\Delta$. We set $S=\Delta \backslash F$ so that $\varphi$ maps $R$ conformally onto $S$.

By the mapping $f \mapsto f \circ \varphi$ the field $M^{\infty}(S)$ is isomorphic to $M^{\infty}(R)$. We consider a valuation $u$ on $M^{\infty}(S)$ given by

$$
u(f)=v(f \circ \varphi) \quad\left(f \in M^{\infty}(S)\right) .
$$

It is certainly a valuation on $M^{\infty}(S)$ and moreover it is also distinguished on $M^{\infty}(S)$ along with $v$ since $\lambda=\lambda \circ \varphi$ for constant (functions) $\lambda \in \mathbf{C}$. Let $b=b_{u}$ be the support of $u$, i.e. $u(I-b)>0$, so that $b \in \bar{S} \subset \bar{\Delta}$ by Proposition 1.

Since $b \in \bar{\Delta}$, either $b \in \partial \Delta$ or $b \in \Delta$. However the former cannot be the case. In fact, if $b \in \partial \Delta$, then, by Proposition 3 , the boundary point $b$ of $S$ have an $H^{\infty}$-barrier on $S$. On the other hand, the last part of Proposition 1 asserts that $b$, having an $H^{\infty}$-barrier on $S$, cannot be the support of any valuation on $M^{\infty}(S)$. This is clearly a contradiction since $b=b_{u}$ is the support of $u$.

Therefore we must have that $b \in \Delta$, and actually $b \in \bar{S} \cap \Delta$. Since $\varphi$ is a conformal mapping of $T$ onto $\Delta$, there exists a unique $c$ in $T$, and actually in $\bar{R} \cap T$, such that $\varphi(c)=b$. The most important point to be stressed here is that $c \notin K$. As a function on $T$ we have $\varphi \in H^{\infty}(T) \subset H^{\infty}(R)$ and

$$
v(\varphi-\varphi(c))=v(I \circ \varphi-b \circ \varphi)=u(I-b)>0 .
$$

Consider the function $\Phi=(\varphi-\varphi(c)) /((I-c) /(I-a))$ which belongs to $H^{\infty}(T)$ $\left(\subset H^{\infty}(R)\right)$ and never vanishes on $T$. The simply connectedness of $T$ then assures the existence of the $n$th root $f_{n}$ of $\Phi$ in $H^{\infty}(T)\left(\subset H^{\infty}(R)\right)$, i.e. $\Phi=f_{n}^{n}$. Thus $v(\Phi)=n v\left(f_{n}\right)$ implies that $v(\Phi)=0$ and $v(I-c)-v(I-a)-v(\varphi-\varphi(c))=$ $-v(\Phi)=0$. Therefore, since $v(I-a)>0$, we have

$$
v(I-c)>v(\varphi-\varphi(c))=u(I-b)>0
$$

and we see that $c=a_{v} \in K$, contradicting $c \notin K$. 
The proof of Theorem 3 is herewith complete.

5.6. Take any arbitrary strictly increasing sequence $\left\{c_{k}\right\}_{1}^{\infty}$ in the interval $(0,1)$ convergent to 1 and an arbitrary sequence $\left\{r_{k}\right\}_{1}^{\infty}$ of positive numbers $r_{k}$ such that $c_{k}+r_{k}<c_{k+1}-r_{k+1}(k=1,2, \ldots)$. Then consider the sequence $\left\{\kappa_{k}\right\}_{1}^{\infty}$ of mutually disjoint closed disks $\kappa_{k}=\Delta\left(c_{k}, r_{k}\right)$ contained in $\Delta=\Delta(0,1)$ and the region (cf. $3.6(2))$

$$
V=\Delta \backslash \bigcup_{k=1}^{\infty} \kappa_{k} .
$$

EXAMPLE 4. The region $V$ in (5.4) is weakly stable.

The proof is immediate in view of Theorem 3. It is an interesting problem to determine whether the above region $V$ of infinite connectivity is stable or not (cf. Open Problems 2 and 3). Let $\alpha_{k}$ be the line segment connecting $1 / k+i$ and $1 / k-i$ $(k \in \mathbf{Z} \backslash\{0\})$ and $\alpha_{\infty}$ the line segment connecting $i$ and $-i$. The region

$$
R=\hat{\mathbf{C}}-\left(\bigcup_{k=1}^{\infty}\left(\alpha_{k} \cup \alpha_{-k}\right)\right) \cup \alpha_{\infty}
$$

is also weakly stable again by Theorem 3 where $\hat{\mathbf{C}}$ is the extended complex plane. We map the simply connected region $R \cap P$ conformally onto the upper half plane $P=\{\operatorname{Im} z>0\}$ by a Riemann mapping function $\psi$ with $\psi(\infty)=\infty$. The Carathéodory theorem on the boundary correspondence assures that $\alpha_{k} \cap \bar{P}$ corresponds to a nondegenerate interval $\beta_{k}$ on the real line $(|\kappa|=1,2, \ldots)$ and $\alpha_{\infty} \cap \bar{P}$ to a single point on the real line, which we can assume to be 0 , by $\psi$. Here we may also assume that $\left\{\beta_{k}\right\}_{1}^{\infty}$ converges to zero decreasingly from the right and $\left\{\beta_{-k}\right\}_{1}^{\infty}$ converges to zero increasingly from the left. By the reflection principle $\psi$ can be continued to a conformal mapping of $R$ onto a Denjoy region (cf. 3.6(3))

$$
S=\hat{\mathbf{C}}-\left(\bigcup_{k=1}^{\infty}\left(\beta_{k} \cup \beta_{-k}\right)\right) \cup\{0\} .
$$

The region $S$ is weakly stable along with $R$ but $\partial S$ contains a degenerate component $\{0\}$. Let $S_{0}=S \cap\{|z|<t\}$ with a sufficiently large $t$ such that the disk $\{|z|<t\}$ contains $\left(\bigcup_{k=1}^{\infty}\left(\beta_{k} \cup \beta_{-k}\right)\right) \cup\{0\}$. Any boundary component of the region $R_{0}=$ $\psi^{-1}\left(S_{0}\right)$ is nondegenerate and thus by Theorem $3 R_{0}$ is weakly stable and so is $S_{0}$. The boundary point $z=0$ is regular for the weakly stable bounded region $S_{0}$ in the sense of the potential theory. To try to construct an $H^{\infty}$-barrier on $S$ at 0 would be a first step to Open Problem 4.

\section{REFERENCES}

1. L. Ahlfors, Open Riemann surfaces and extremal problems on compact subregions, Comment. Math. Helv. 24 (1950), 100-134.

2. N. Alling, The valuation theory of meromorphic function fields, Proc. Sympos. Pure Math., vol. 11, Amer. Math. Soc., Providence, R.I., 1968, pp. 8-29.

3. O. Endler, Valuation theory, Springer, 1972.

4. F. Forelli, private communication.

5. B. Fuglede, Sur les fonctions finement holomorphes, Ann. Inst. Fourier (Grenoble) 31 (1981), $57-88$. 
6. J. Garnett and P. Jones, The corona theorem for Denjoy domains, Acta Math. 155 (1985), 31-40.

7. M. Heins, Algebraic structure and conformal mapping, Trans. Amer. Math. Soc. 89 (1958), 267-276.

8. _ Complex function theory, Academic Press, 1968.

9. H. Iss'ssa, On meromorphic function fields on a Stein variety, Ann. of Math. (2) 83 (1966), $34-46$.

10. S. Lang, Algebra, Addison-Wesley, 1965.

11. H. Royden, Rings of analytic and meromorphic functions, Trans. Amer. Math. Soc. 83 (1956), 269-276.

12. __ Rings of meromorphic functions, Proc. Amer. Math. Soc. 9 (1958), 959-965.

13. __ Algebras of bounded analytic functions on Riemann surfaces, Acta Math. 114 (1965), 113-142.

14. L. Sario and M. Nakai, Classification theory of Riemann surfaces, Springer, 1970.

15. O. Schilling, The theory of valuations, Math. Surveys, no. 4, Amer. Math. Soc., Providence, R.I., 1950.

16. M. Tsuji, Potential theory in modern function theory, Chelsea, New York, 1975.

17. L. Zalcman, Bounded analytic functions on domains of infinite connectivity, Trans. Amer. Math. Soc. 114 (1969), 241-269.

18. O. Zariski and P. Samuel, Commutative algebra, Vol. 2, Van Nostrand, 1960.

Department of Mathematics, Nagoya instutite of Technology, Gokiso, SHOWA, NAGOYA 466 , JAPAN 